\title{
ECOLOGIE D'UNE POPULATION DE VANDOISES, LEUCISCUS LEUCISCUS (L.) DANS LA RIVIERE OURTHE (BASSIN DE LA MEUSE, BELGIQUE)
}

\author{
par J. C. PHILIPPART ${ }^{1}$
}

A partir d'échantillons de vandoises capturés par pêche à l'électricité de 1971 à 1976, les principales caractéristiques de la population de l'Ourthe onl été décrites et analysées : structure par âge (longévité maximale : 15 ans), variations de l'abondance des classes d'âge 1958-1972, taux annuel de mortalité ( $M=38 \%$ ), taux de croissance en longueur (21 $\mathrm{cm}$ à 5 ans pour les deux sexes réunis;, sex ratio mâles/femelles : 1,04$)$, âge de la maturité sexuelle $(3-4$ ans et $16 \mathrm{~cm}$ ), fécondité absolue individuelle en fonction de la taille et de l'âge, cycle saisonnier de la condition et du développement des gonades, relations poids-longueur, période de ponte. La dynamique (croissance pondérale, survie, fécondité et production somatique) d'une cohorte type de vandoises est établie. Des estimations sont proposées pour la biomasse du peuplement $(6,3 \mathrm{~kg} / \mathrm{ha})$, la production somatique $(2,3 \mathrm{~kg} / \mathrm{ha} / \mathrm{an})$, le potentiel reproducteur $\left(35 \mathrm{ovules} / \mathrm{m}^{2}\right)$ et l'exploitation par la péche $(2,0 \mathrm{~kg} / \mathrm{ha} / \mathrm{an})$. La discussion traite de la démographie de la vandoise dans quelques rivières européennes.

\section{Population ecology of the dace, Leuciscus leuciscus (L.), in the river Ourthe (River Meuse Basin, Belgium).}

From samples of dace taken by electrofishing from 1971 to 1976, the chief features of the population have been described and analysed: age structure (maximum age was 15 years), variations in the abundance of the different yearclasses for 1958-1972, annual mortality rate $(M=38 \%)$, growth rate in length $(21 \mathrm{~cm}$ at 5 years for the two sexes combined), sex ratio (males/females $=1.04$ ), age of sexual maturity (3-4 years and $16 \mathrm{~cm}$ ), absolute individual fecundity as a function of size and age, seasonal cycle of the condition and development of the gonads, length-weight relationship, spawning period. The dynamics (growth in weight, survival, fecundity and somatic production) are summarised for a typical cohort. Estimates are made for the absolute population biomass $(6.3 \mathrm{~kg} / \mathrm{ha})$, somatic production $(2.3 \mathrm{~kg} / \mathrm{ha} /$ year $)$, potential reproduction $\left(35\right.$ ovules $\left./ \mathrm{m}^{2}\right)$ and the exploitation of the fishery $(2.0 \mathrm{~kg} / \mathrm{ha} / \mathrm{year})$. The discussion reviews the demography of dace in several european rivers.

\section{INTRODUCTION}

La vandoise, Leuciscus leuciscus (L.), est un Poisson Cyprinidae largement distribué dans les eaux douces européennes (Blanc et coll. 1971). Son écologie a été étudiée en Europe de l'Est (Klimczyk 1966,

1. Chercheur qualifié du Fonds National de la Recherche Scientifique (F.N.R.S.). Institut de Zoologie de l'Université de Liège. Service d'Ethologie et Psychologie animales. Aquarium (Prof. J. C. RUwET). 22, quai Van Beneden, B 4020 Liège, Belgique. 
Penczak 1967, Penczak et coll. 1978, Klimczyk-Janikowska 1971) et surtout dans les Iles Britanniques (Williams 1965, 1967, Mackay et Mann 1969, Cragg-Hine et Jones 1969, Kennedy 1969, Mathews 1971, Mathews et Williams 1972, Hart et Pitcher 1973, Mann 1974, Hellawell 1974 a, b, Starkie 1976, Wilkinson et Jones 1977, Mills 1980), mais il y a peu de données pour l'Europe occidentale continentale (Bourgeois 1963, Philippart 1971, Hofstede 1974).

Cet article concerne l'écologie des populations de vandoises dans l'Ourthe, le principal affluent de la Meuse en Belgique. L'étude a été réalisée de 1971 à 1976 dans un tronçon de rivière de $20 \mathrm{~km}$ ( fıg. 1) correspondant à une "Zone à Barbeau » selon Huet (1949); des descriptions détaillées du milieu sont données dans plusieurs publications antérieures (Micha 1970, 1971, Philippart 1977, 1979 b).

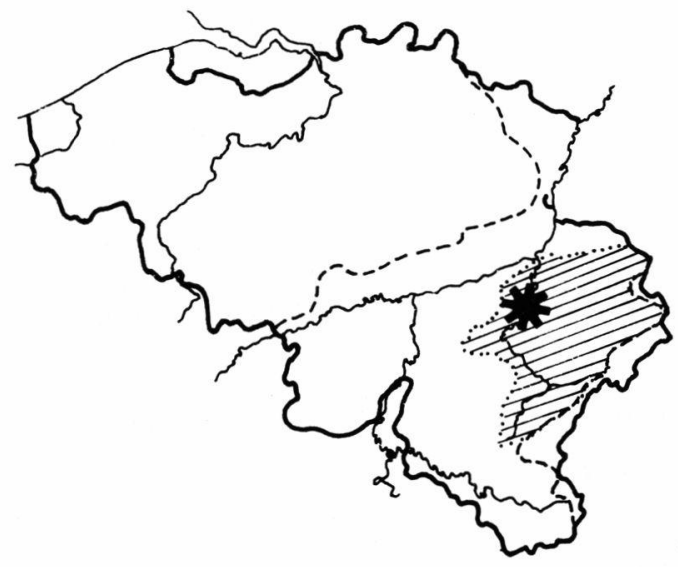

Fig. 1. - Carte de localisation de l'Ourthe et du secteur étudié.

Cette étude s'intègre dans une recherche sur l'écologie et la démographie comparée des Cyprinidae rhéophiles lithophiles, Barbus barbus, Chondrostoma nasus (Philippart, sous presse) et Leuciscus cephalus (Philippart 1981).

\section{1. - MATERIEL et METHODES}

Les vandoises étaient capturées par la technique de pêche à l'électricité (courant continu 200-300 V) lors d'échantillonnages organisés pendant toute l'année (surtout en juin-novembre) dans 90 secteurshabitats longs de $220 \mathrm{~m}$ en moyenne. Les poissons étaient systématiquement mesurés (longueur au creux de la nageoire caudale) et ćventuellement pesés sous-échantillonnage) ou sexés (en période de reproduction). Des écailles étaient prélevées dans les deux premières 


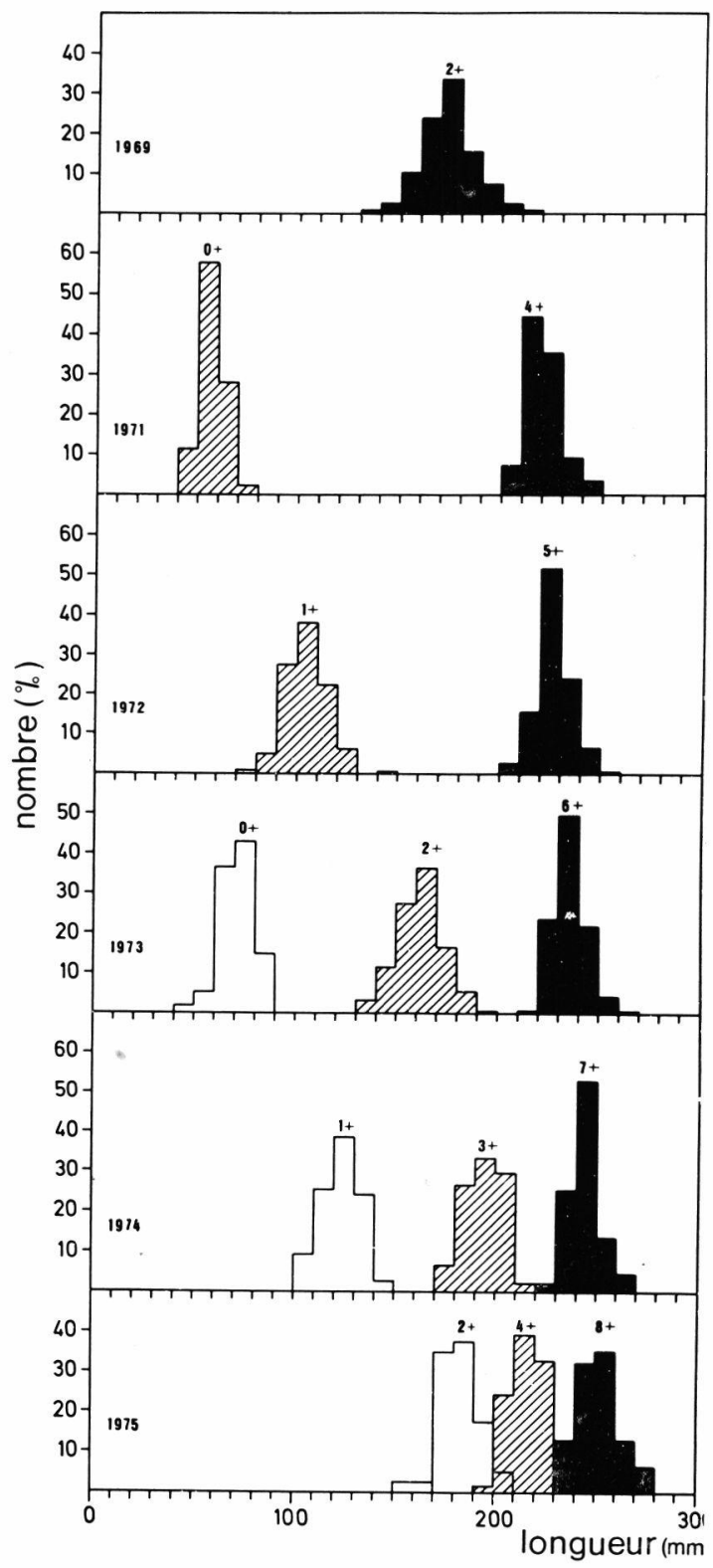

Fig. 2. - Structure par taille et validité des critères d'âge chez la vanduise de l'Ourthe. Croissance des classes d'âge 1971 (hachuré) et 1973 (blanc) à partir du groupe $0+$ et de la classe d'âge 1967 (noir) à partir du groupe $2+$. A clıaque déplacement annuel des modes correspond l'addition d'un annulus sur les écailles et les os operculaires. 
rangées, au-dessus de la ligne latérale, à hauteur de l'anus (Philippart 1971). Aux différentes périodes de l'année, des échantillons de poissons furent prélevés en vue des études au laboratoire selon les méthodes classiques (Ricker 1968) : prélèvement des os operculaires, détermination du sexe et du stade de maturation sexuelle, pesée des ovaires et testicules, prélèvement et conservation des ovaires pour le comptage des ovules. Sur le terrain, tous les poissons étaient remis à l'eau après avoir reçu un marquage de groupe (mutilation des nageoires ; tatouage au dermojet Hart et Pitcher 1969) pour les essais de dénombrement par marquage-recapture.

L'âge est déterminé par la méthode Petersen (fig. 2) et par la méthode scalimétrique (écailles et os operculaires) en utilisant les critères d'âge définis par divers auteurs (Crag-Hines et Jones 1969 , Philippart 1971, Mathews et Williams 1972, Hellawell 1974 a, b, Mann 1974). Les courbes de croissance annuelles en longueur sont établies en calculant la taille moyenne des groupes d'âge à la fin de la periode de croissance (regroupement des poissons capturés en septembremars) ou par la technique de rétro-mesure des écailles (relations corps-écaille: $\log$ longueur du corps $(\mathrm{mm})=0,58+0,63$ Log radius antérieur de l'écaille $(\mathrm{mm} \times 20)$; formule de calcul de Le Cren (1947). Les relations poids-longueur sont calculées par les méthodes ciassiques (Le Cren 1951) pour les mâles, femelles et sexes réunis à quatre périodes de l'année: janvier-mars (avant la ponte), mars (après la ponte) juin, juillet-septembre et octobre-décembre. Les paramètres de mortalité-survie sont évalués à partir des structures par âge annuelles 1971-1976 (Tableau I) en appliquant la méthode de Kempe (1962). La fécondité individuelle est estimée par comptage des ovules chez des vandoises capturées entre octobre et fin mars.

Dans l'étude de l'évolution d'une cohorte type, les résultats sont exprimés en terme de biomasse et de production par recrue à 2 ans (Backiel 1971). La production (P) selon Chapman (1968) est calculée par la méthode arithmétique de Ricker (1946): $P=G . \bar{B}$. G est le coefficient instantané de croissance pondérale sur une base annuelle: $\mathrm{G}=\ln \mathrm{pm} 2-\ln \mathrm{pm} 1$ où $\mathrm{pm} 1$ et $\mathrm{pm} 2$ représentent les poids moyens (d'après une équation de Von Bertalanffy) de la cohorte aux âges 1 et 2 en années. $\bar{B}$ est la biomasse moyenne $\left(\frac{B(1)+B(f)}{2}\right)$ de la cohorte entre le début de la croissance annuelle fixée au 15 mai $(B 1=\mathrm{N} 1 \times$ $\mathrm{pm} 1)$ et la fin de cette croissance fixée au 15 septembre $(\mathrm{Bf}=\mathrm{Nf} \times$ pm2): les effectifs de la cohorte en septembre (Nf) sont estimés à partir des effectifs annuels théoriques initiaux (N1) en mai, en supposant (Backiel 1971) un taux instantané de mortalité (Z) constant au cours de l'année et valant donc de mai à septembre $5 / 12$ de $Z$ annuel (d'où $\mathrm{Nf}=\mathrm{N} 1 \mathrm{e}^{-0,42} \mathrm{Z}$ ). Les rapports production/biomasse sont 
calculés en faisant intervenir la biomasse moyenne annuelle de la cohorte c'est-à-dire : $\bar{B}=\frac{\mathrm{B} 1+\mathrm{B} 2}{2}$ avec $\mathrm{B}=\mathrm{N} 1 \times \mathrm{pm} 1, \mathrm{~B} 2=\mathrm{N} 2 \times$ $\mathrm{pm} 2$ et $\mathrm{N} 2=\mathrm{N} 1 \mathrm{e}^{-\mathrm{Z}}$.

\section{2. - RESUltAtS}

\subsection{Longévité et croissance en longueur}

Tableau I. - Structure par âge des échantillons de vandoises (sexes réunis) capturés dans l'Ourthe de 1971 à 1976. L'abondance relative des groupes d'âge est exprimée en \% du nombre total (n) de poissons capturés.

\begin{tabular}{ccccccc}
\hline $\begin{array}{c}\text { Groupes } \\
\text { d'âge }\end{array}$ & $\begin{array}{c}71-72 \\
(\mathrm{n}=84)\end{array}$ & $\begin{array}{c}72-73 \\
(\mathrm{n}=521)\end{array}$ & $\begin{array}{c}\text { Abondance relative }(\%) \\
(\mathrm{n}=727)\end{array}$ & $\begin{array}{c}74-75 \\
(\mathrm{n}=336)\end{array}$ & $\begin{array}{c}75-76 \\
(\mathrm{n}=167)\end{array}$ & $\begin{array}{c}\text { Moyenne } \\
\end{array}$ \\
\hline $1+$ & 8,3 & 46,4 & 1,1 & 22,3 & 2,4 & 16,1 \\
$2+$ & 4,8 & 1,3 & 61,2 & 0,9 & 24,0 & 18,4 \\
$3+$ & 4,8 & 2,5 & 1,4 & 41,3 & 1,8 & 10,4 \\
$4+$ & $\mathbf{6 4 , 2}$ & 3,6 & 0,4 & 0,6 & 47,2 & 23,2 \\
$5+$ & - & 39,2 & 3,6 & 2,7 & 0,6 & 9,2 \\
$6+$ & - & 0,8 & 27,0 & 25,9 & 0,6 & 6,2 \\
$7+$ & $\mathbf{1 5 , 5}$ & 1,2 & 0,6 & 0,6 & 3,0 & 9,2 \\
$8+$ & - & 3,6 & 1,8 & 0,6 & 18,6 & 4,9 \\
$9+$ & - & 0,2 & 1,9 & 1,8 & - & 0,5 \\
$10+$ & - & 0,2 & 0,5 & - & - & 0,5 \\
$11+$ & - & 0,2 & - & - & 1,8 & 0,4 \\
$12+$ & 2,4 & - & - & - & - & 0,5 \\
$13+$ & - & 0,8 & - & 0,3 & - & 0,2 \\
$14+$ & - & - & 0,5 & - & - & 0,1 \\
$15+$ & - & - & - & 0,3 & - & 0,3 \\
\hline
\end{tabular}

La longévité (Tableau I) et la taille maximale observées sont respectivement $15+(q$ de $275 \mathrm{~mm})$ et $288 \mathrm{~mm}(q 13+)$. Les mâles grandissent un peu plus rapidement que les femelles (Tableau II) mais les différences ne sont jamais statistiquement significatives $(P>0,05)$. L'expression la plus générale de la croissance linéaire moyenne des deux sexes réunis est une équation de Von Bertallanffy (cf. Rıcker 1968) avec les paramètres $L \infty=270 \mathrm{~mm}, K=0,33$ et $t_{o}=0,24$.

La vandoise se caractérise par une croissance initiale assez rapide puisque la taille moyenne à 2 ans représente $40 \%$ de la taille limite $\mathrm{L} \infty=270 \mathrm{~mm}$ (à \pm 15 ans). Au cours d'un cycle annuel, la croissance linéaire a lieu de mai à septembre, quand la température de l'eau dépasse $11-12^{\circ} \mathrm{C}$. Les variations annuelles de la température moyenne 
de l'eau pendant les mois de croissance active (juin-juillet-août) entraînent des fluctuations de la croissance annuelle moyenne relative de la population (Tableau III). Les croissances les plus faibles s'obsevent pendant les années "froides", 1965 et 1972 ; la relation température-croissance est moins nette pour les années à croissance rapide.

Tableau II. - Longueur moyenne ( $\mathrm{L}$ en $\mathrm{mm}$ ) en fonction de l'âge chez les vandoises de l'Ourthe.

Mâles et femelles: longueur moyenne des groupes d'âge à la fin de l'année de croissance (rétro-mesure à partir des écailles pour 1 an).

Sexes réunis: longueur moyenne des groupes d'âge pour l'ensemble des captures (individus sexés et non sexés).

\begin{tabular}{|c|c|c|c|c|c|c|c|c|}
\hline \multirow{2}{*}{$\begin{array}{c}\text { Age } \\
\text { (années) }\end{array}$} & \multicolumn{3}{|c|}{$\begin{array}{l}\text { Mâles } \\
(\mathrm{n}=246)\end{array}$} & \multicolumn{3}{|c|}{$\begin{array}{l}\text { Femelles } \\
(n=262)\end{array}$} & \multicolumn{2}{|c|}{$\begin{array}{c}\text { Sexes réunis } \\
(\mathrm{n}=2797)\end{array}$} \\
\hline & $\mathrm{n}$ & $\bar{L}$ & $\begin{array}{l}\text { I.C. } \\
95 \%\end{array}$ & $\mathrm{n}$ & $\mathrm{L}$ & $\begin{array}{l}\text { I.C. } \\
95 \%\end{array}$ & $\mathrm{n}$ & $\mathrm{L}$ \\
\hline I & 95 & 69 & \pm 2 & 222 & 66 & \pm 2 & 1032 & 57 \\
\hline II & 36 & 113 & \pm 3 & 44 & 107 & \pm 3 & 328 & 109 \\
\hline III & 41 & 167 & \pm 3 & 42 & 164 & \pm 3 & 492 & 164 \\
\hline IV & 12 & 200 & \pm 6 & 12 & 195 & \pm 6 & 163 & 196 \\
\hline $\mathrm{V}$ & 20 & 218 & \pm 4 & 23 & 215 & \pm 4 & 150 & 217 \\
\hline VI & 89 & 228 & \pm 2 & 83 & 227 & \pm 2 & 222 & 227 \\
\hline VII & 13 & 235 & \pm 6 & 18 & 238 & \pm 6 & 204 & 236 \\
\hline VIII & 8 & 254 & \pm 6 & 12 & 245 & \pm 6 & 111 & 245 \\
\hline IX & 17 & 258 & \pm 5 & 17 & 255 & \pm 5 & 64 & 254 \\
\hline $\mathrm{X}$ & 4 & 251 & & 3 & 259 & & 9 & 257 \\
\hline $\mathrm{XI}$ & 3 & 262 & & - & & & 8 & 260 \\
\hline XII & 1 & 263 & & 1 & 280 & & 4 & 265 \\
\hline XIII & - & & & 2 & 279 & & 2 & 279 \\
\hline XIV & 1 & 278 & & 4 & 279 & & 5 & 279 \\
\hline $\mathrm{XV}$ & 1 & 271 & & 1 & 273 & & 2 & 272 \\
\hline XVI & - & & & 1 & 275 & & 1 & 275 \\
\hline
\end{tabular}

\subsection{Structure par âge et variations annuelles du recrutement}

La structure par âge de la population (Tableau I) est irrégulière et même discontinue à cause de fortes variations annuelles du succès de la reproduction et du recrutement des classes d'âge. Des inclices d'abondance relative des classes d'âge 1958-1972 sont présentés dans le tableau III : il apparaît quatre classes d'âge dominantes $(1959,1964$, 1967 et 1971) et une majorité de classes d'âge peu importantes (I.A. $<30$ ).

L'abondance des classes d'âge est corrélée négativement $(r=-0,70$; $\mathbf{P}<0,01 ; 13 \mathrm{~d}$. 1.) avec les précipitations sur le bassin et positivement $(\mathrm{r}=+0,67 ; \mathrm{P}<0,01 ; 13 \mathrm{~d} .1$.$) avec la température moyenne régionale$ de l'air (prise comme indice de la température de l'eau) en juin-juilletaoût. Les quatre classes d'âge dominantes sont associées à des conditions climatiques favorables, non seulement en été (précipitations 
$<250 \mathrm{~mm}$; température moyenne de l'air $>17,2^{\circ} \mathrm{C}$ ) mais également pendant la période de reproduction (débit moyen $<15 \mathrm{~m}^{3} / \mathrm{sec}$ et relativement constant en mars, avril et mai). Le système de détermination climatique du succès du recrutement se schématise donc comme suit :

$\begin{array}{ccc}\begin{array}{c}\text { Climat } \\ \text { en mars - avril - mai (débit) }\end{array} & \begin{array}{l}\text { Climat en juin - juillet - août } \\ \text { (température, précipitations) }\end{array} & \text { Recrutement } \\ \text { bon } & \text { bon } & \text { important } \\ & \text { mauvais } & \text { faible } \\ \text { mauvais } & \text { bon } & \text { faible } \\ & \text { mauvais } & \text { faible }\end{array}$

Quand une conjonction de conditions climatiques favorables permet un recrutement d'une certaine importance, l'abondance des classes d'âge (I.A.) est proportionnelle à la température estivale moyenne $(T)$ : I.A. $=-2003+127 \mathrm{~T}\left(\mathrm{n}=4 ; \mathrm{r}^{2}=0,96\right)$.

Au moment de la reproduction, en fin mars - début avril, et pendant la longue phase d'incubation des œufs (25-30 jours à $11-13^{\circ} \mathrm{C}$ d'après Bourgeois 1963 et Kennedy 1969 ; 30 jours dans la R. Frome d'après Mills 1980), les facteurs climatiques agiraient sur le déroulement normal de la ponte (caractères inadéquats des frayères en cas de débit excessif́ par exemple) ou/et sur la survie des embryons et des larves: destruction mécanique du frai par les hauts débits, envasement des frayères, perturbation de l'incubation et de l'éclosion par des températures trop basses ( $<6^{\circ}$ d'après Mills 1980). En juin-août, la relation entre le succès du recrutement et le climat proviendrait d'une action positive de la température de l'eau sur le taux de croissance des alevins (cf. Broughton et Jones 1978 pour Rutilus rutilus) et sur leur taille à la fin de l'année de croissance et pendant l'hiver; une croissance plus rapide des alevins augmenterait leurs chances de survie et par conséquent l'importance du recrutement de la classe d'âge concernée.

\subsection{Taux annuel moyen de mortalité totale}

La courbe de capture (fig. 3 ) construite à partir des structures par âge moyennes du tableau III permet de calculer un taux annuel moyen de mortalité totale, $M=30 \%$ (coefficient instantané de mortalité $\mathrm{Z}=0,48)$ pour les mâles et femelles $\geqslant 4$ ans. D'après les observations de Mann (1974), ce taux de mortalité peut raisonnablement s'appliquer aux vandoises de 2 ans $(1+)$ et plus. Le taux de mortalité est certainement plus élevé dans les groupes d'âge $0+$ et $1+$ (Mathews 1971) mais aucune estimation n'est disponible actuellement. Il ne semble pas exister de différence significative entre les taux annuels moyens de mortalité des mâles et femelles (cf. sex ratio). 


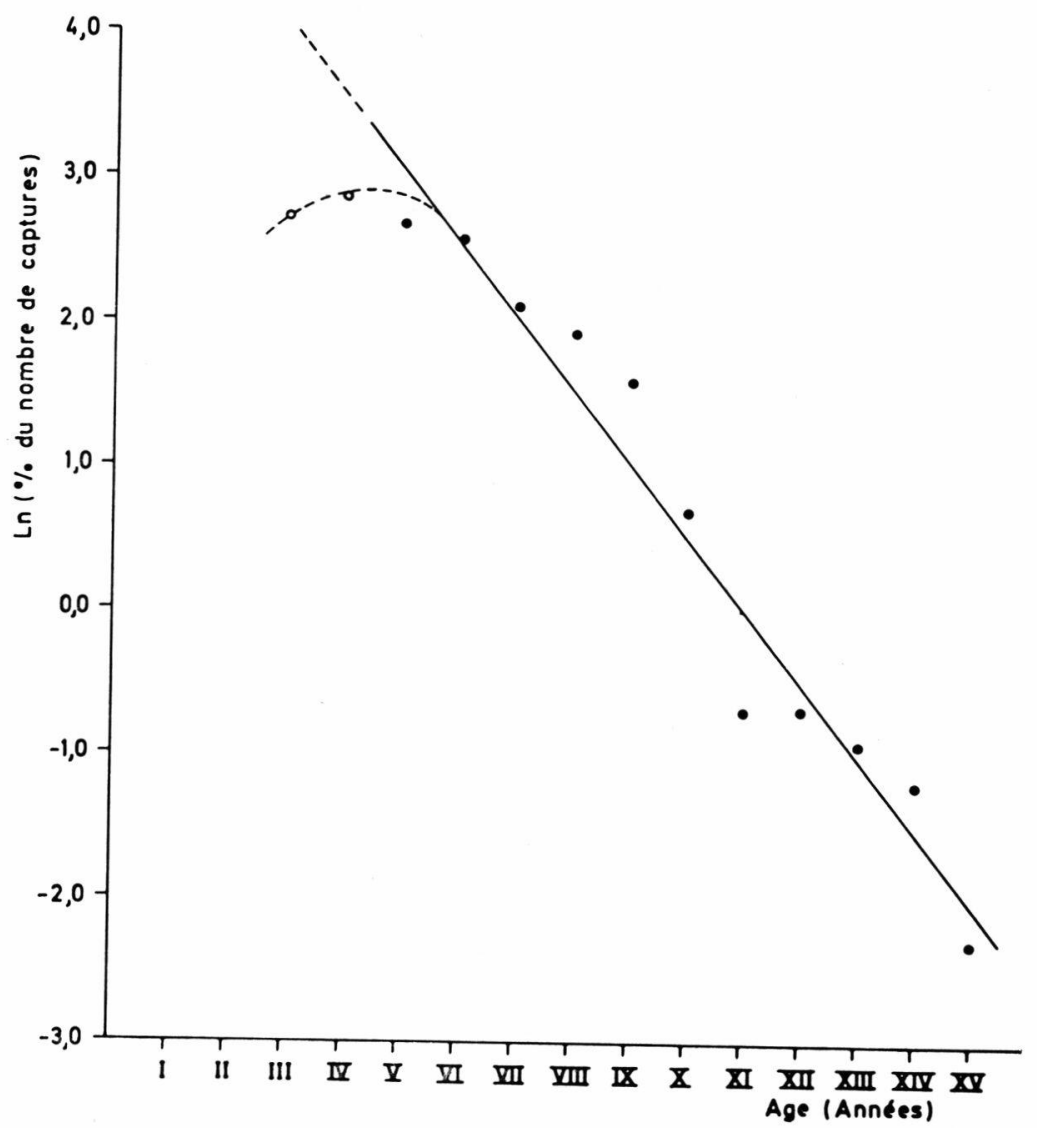

Fig. 3. - Courbe de capture logarithmique (ln) pour les vandoises (sexes réunis) de l'Ourthe. Les points représentent les \% moyens $71-76 \mathrm{du}$ tableau I ajustés (moyennes mobiles sur trois groupes d'âge). Le dôme de la courbe (en pointillé) indique la zone de sélectivité de la méthode de capture.

Le cycle saisonnier de la mortalité n'a pas été déterminé mais les adultes subissent une mortalité importante en avril-mai après la reprocuuction. Malgré le faible intérêt porté par les pêcheurs à cette espèce, l'effet de la pêche est sensible vu l'absence de taille légale de capture et le fait que les vandoises sont souvent capturées en pêchant d'autres espèces.

Pendant la saison de pêche de 1974 (juin à septembre) les prises de vandoises ont été évaluées à 14 individus et $2 \mathrm{~kg} / \mathrm{ha}$ (Philippart 1979 a), soit approximativement $20 \%$ des effectifs et $32 \%$ de la biomasse dénombrés en fin 1973. La mortalité par la pêche touche principalement les poissons de plus de $20 \mathrm{~cm}(\geqslant 100 \mathrm{~g})$. 
TABLEAU III. - Croissance annuelle relative et abondance relative des classes d'âge chez les vandoises de l'Ourthe de 1958 à 1975. Indices de croissance et d'abondance calculés par la méthode de Kempe (1962).

(1) indices calculés à partir des résultats de la rétro-mesure des écailles ( $\mathrm{n}=$ 816 données).

(2) lame d'eau précipitée en juin - juillet - août sur le bassin de l'Ourthe limité à la région étudiée.

(3) température moyenne de l'air en juin - juillet - août à la station météorologique nationale d'Uccle.

\begin{tabular}{|c|c|c|c|c|}
\hline $\begin{array}{l}\text { Années - } \\
\text { classes } \\
\text { d'âge }\end{array}$ & $\begin{array}{c}\text { Indices } \\
\text { de } \\
\text { croissance } \\
\text { annuelle (1) } \\
\text { I.C. } \\
\end{array}$ & $\begin{array}{c}\text { Indices } \\
\text { d'abondance } \\
\text { des } \\
\text { classes d'âge } \\
\text { I.A. } \\
\end{array}$ & $\begin{array}{l}\text { Précipitations } \\
\text { estivales (2) } \\
\text { (mm) }\end{array}$ & $\begin{array}{c}\text { Température } \\
\text { moyenne } \\
\text { estivale } \\
\text { de l'air (3) } \\
\left({ }^{\circ} \mathrm{C}\right)\end{array}$ \\
\hline 1958 & - & 29 & 308 & 17,5 \\
\hline 1959 & - & 471 & 173 & 19,6 \\
\hline 1960 & - & 1 & 337 & 17,2 \\
\hline 1961 & - & 29 & 288 & 17,3 \\
\hline 1962 & - & 10 & 220 & 16,5 \\
\hline 1963 & - & 10 & 239 & 17,2 \\
\hline 1964 & 108 & 159 & 190 & 17,2 \\
\hline 1965 & 67 & 17 & 312 & 16,1 \\
\hline 1966 & (119) & 7 & 381 & 17,2 \\
\hline 1967 & 120 & 332 & 159 & 18,1 \\
\hline 1968 & 99 & 30 & 272 & 17,1 \\
\hline 1969 & 106 & 13 & 304 & 18,2 \\
\hline 1970 & 98 & 6 & 241 & 18,4 \\
\hline 1971 & 110 & 263 & 241 & 17,9 \\
\hline 1972 & 77 & 17 & 256 & 15,9 \\
\hline 1973 & 117 & - & 165 & 18,9 \\
\hline 1974 & 89 & - & 266 & 16,1 \\
\hline 1975 & 107 & - & 180 & 18,0 \\
\hline
\end{tabular}

\subsection{Biologie de la reproduction et fécondité}

TableaU IV. - Sex ratio en fonction de l'âge chez les vandoises de l'Ourthe.

\begin{tabular}{|c|c|c|c|}
\hline \multirow{2}{*}{$\frac{\text { Age }}{\text { (années) }}$} & \multicolumn{3}{|c|}{ Nombre de poissons } \\
\hline & 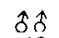 & 99 & $\hat{\beta} \hat{\delta} / \phi 9$ \\
\hline II & 42 & 51 & $0,82: 1$ \\
\hline III & 68 & 46 & $1,48: 1$ \\
\hline IV & 15 & 15 & $1,00: 1$ \\
\hline V & 22 & 23 & $0,96: 1$ \\
\hline VI & 97 & 83 & $1,17: 1$ \\
\hline VII & 15 & 24 & $0,63: 1$ \\
\hline VIII & 10 & 12 & $0,83: 1$ \\
\hline IX & 17 & 19 & $0,89: 1$ \\
\hline $\mathrm{X}$ & 4 & 3 & $1,33: 1$ \\
\hline$X I$ & 3 & 0 & - \\
\hline XII & 1 & 1 & $1,00: 1$ \\
\hline XIII & 0 & 2 & $\bar{\sigma}$ \\
\hline XIV & 1 & 4 & $0,25: 1$ \\
\hline XV & 1 & 1 & $1,00: 1$ \\
\hline Total & 296 & 284 & $1,04: 1$ \\
\hline
\end{tabular}


Le sex ratio général de la population (poissons $\geqslant 1+$ ) est équilibré (Tableau IV). Par rapport aux groupes d'âge $2-5$ ans $(\hat{\jmath} \hat{\delta} / \not q+=1,09: 1)$ et $6-10$ ans $(\hat{\delta} \delta / \% \circ=1,04: 1)$, on note une légère prédominance des femelles dans les groupes d'âge $\geqslant 10$ ans ( $\hat{\delta} \hat{\delta} / q 0$ : $0,88: 1$ ), mais cette différence est biologiquement peu significative à cause du petit nombre d'individus présents dans l'échantillon. Les vandoises de l'Ourthe deviennent sexuellement mûres pendant leur troisième année ( $84 \%$ des mâles et $71 \%$ des femelles dans le groupe $1+$, à une taille moyenne de $\pm 16 \mathrm{~cm})$ et leur quatrième année de vie (100\% des mâles et femelles mûrs).
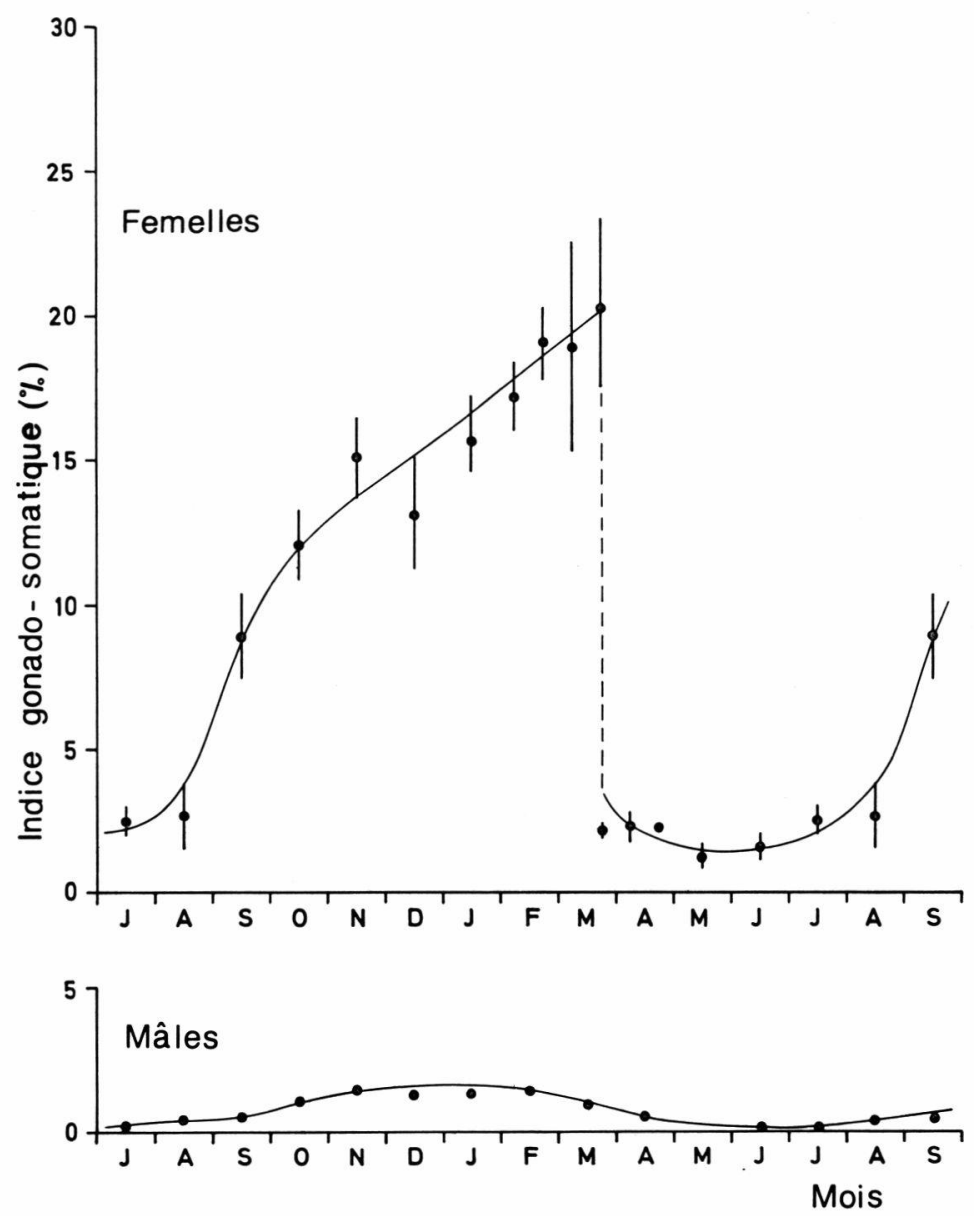

Fig. 4. - Cycle saisonnier de l'indice gonado-somatique moyen (I.G.S. = poids des gonades en \% du poids corporel) chez les vandoises mâles $(M ; n=112)$ et femelles $(\mathrm{F} ; \mathrm{n}=147)$ de la classe d'âge 1967 en 1972-1973 $(5+-6+)$. Traits verticaux $=$ I.C. $95 \%$ des moyennes mensuelles ou bihebdomadaires. 
Le cycle annuel de la croissance des gonades apparaît sur la fig. 4. A leur stade maximum de développement, les testicules représentent en moyenne 2-3\% du poids corporel total et les ovaires $13 \%$ ou $20 \%$ selon qu'il s'agit de femelles dans leur première année de maturité $(2+)$ ou plus âgées $(\geqslant 3+)$. Les ovaires contiennent, en plus des ovocytes de remplacement, un seul type d'ovules (ovogénèse synchrone). Au moment de la ponte, presque toute la masse de ceux-ci est expulsée, ce qui entraîne une forte diminution de la condition (Tableau V) ; ce phénomène est moins marqué chez les mâles dont la

Tableau V. - Relations poids - longueur et cycle de la condition (poids moyen à 22 ou $12 \mathrm{~cm})$ chez les vandoises $(>0+)$ de l'Ourthe.

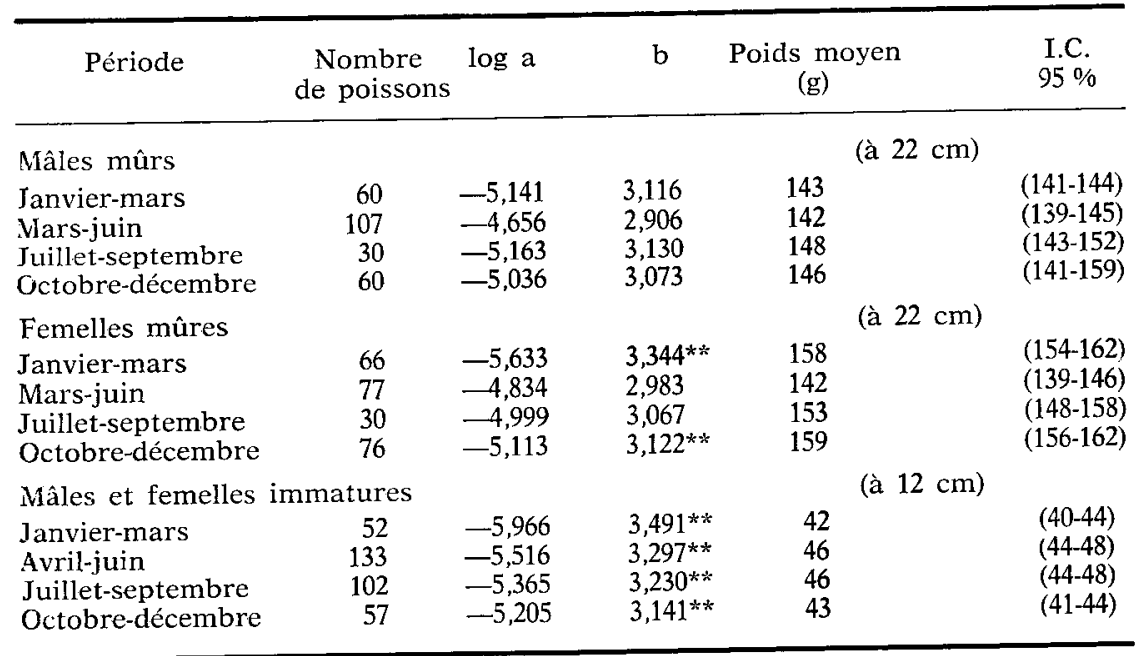

$* * b \neq 3 ; P<0,01$.

prcduction sexuelle est peu abondante et dont l'expulsion de la laitance s'étale sur plusieurs semaines. Dans l'Ourthe, la vandoise pond en fin mars-début avril (en même temps que l'ombre Thymallus thymallus (L.) et un peu avant le hotu, Chondrostoma nasus (L.)) quand l'eau se réchauffe jusqu'à $7-9^{\circ} \mathrm{C}$. Les œufs sont déposés sur les graviers dans les courants peu profonds (Kennedy 1969); Balon (1975) classe la vandoise dans un groupe (espèce phytolithophile) intermédiaire entre les espèces typiquement lithophiles ou phytophiles.

La fécondité absolue individuelle ( $\mathrm{Fa}$ ) des femelles est une fonction $\log$ linéaire de la taille ( $\mathrm{L}$ en $\mathrm{mm}$ ) :

$$
\log \mathrm{Fa}=-3,64+3,28 \log \mathrm{L}, \mathrm{n}=105, \mathrm{r}^{2}=0,94 \text {. }
$$

Une vandoise de $20 \mathrm{~cm}$ produit environ 8100 ovules. La fécondité augmente linéairement avec l'âge jusqu'à 8 ans (taille moyenne 


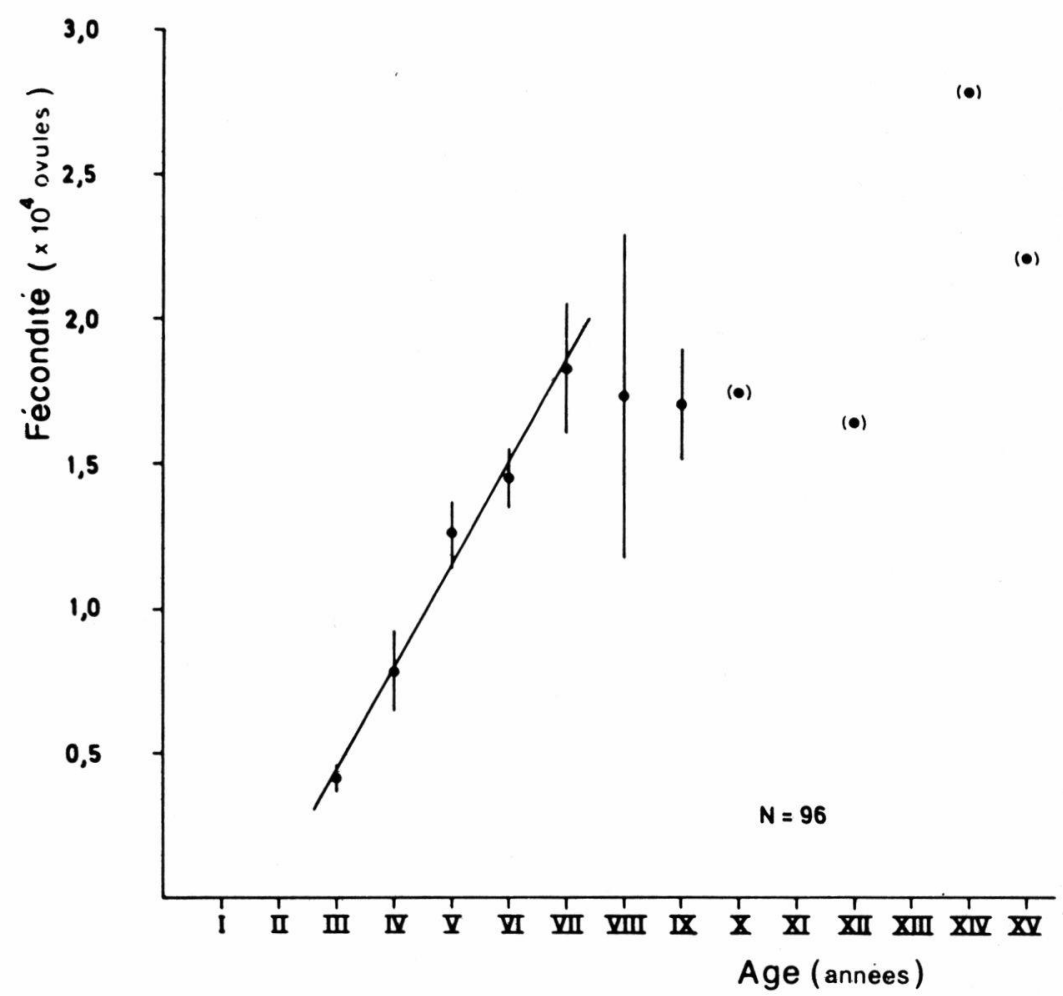

Fitc. 5. - Fécondité absolue ( $\mathrm{Fa}$ ) des vandoises de l'Ourthe en fonction de l'âge (A). Traits verticaux : I.C. $95 \%$ de la fécondité moyenne/âge. (๑) 1 seul individu. Pour les âges III-VII, l'équation de la droite de régression est : $\mathrm{Fa}=3572 \mathrm{~A}$ $6314, \mathrm{n}=5, \mathrm{r}^{2}=0,97$. Les fécondités extrêmes observées sont un maximum de 27800 ovules chez une femelle de $288 \mathrm{~mm}$ et $13+$ et un minimum de 2900 ovules chez une femelle de $160 \mathrm{~mm}$ et $2+$.

$\pm 25 \mathrm{~cm}$ ) puis la relation devient imprécise à cause du nombre insuffisant de poissons dans l'échantillon (fig. 5); on remarque néanmoins une tendance à l'infléchissement de la courbe fécondité/âge avec l'âge, ce qui concorde avec les observations de Wilkinson et Jones (1977) sur les vandoises de l'Emral Brook au Pays de Galles. Dans l'Ourthe, ce phénomène résulte en grande partie d'une augmentation de la taille et du poids des ovules avec la taille et l'âge du poisson : 1 gramme d'ovaire contient en moyenne 413 ovules chez les femelles $\geqslant 4+$ $(>200 \mathrm{~mm})$ et 604 ovules chez les femelles $2+-3+(<200 \mathrm{~mm})$; dans les échantillons de novembre-janvier, la relation entre le nombre d'ovules/gramme d'ovaire ( $\mathrm{N}$ ov/g) et la taille corporelle ( $\mathrm{L}$ en $\mathrm{mm}$ ) est : $\mathrm{N}$ ov $/ \mathrm{g}=1059-23 \mathrm{~L}\left(\mathrm{n}=50, \mathrm{r}^{2}=0,92\right)$, mais un effet de sénilité semble également jouer. Sur ce point, signalons que nous n'avons pas observé, contrairement à Wilkinson et Jones (op. cit.), une aug- 
mentation avec l'âge de la proportion des vandoises présentant une atrophie folliculaire complète.

La fécondité relative des vandoises de l'Ourthe est d'environ 78000 ovules $/ \mathrm{kg}$ de poids corporel 2 à 4 semaines avant la ponte; les ovules pèsent en moyenne $2,3 \mathrm{mg}$. Mais ces deux paramètres varient (augmentation de la taille des ovules et diminution de la fécondité relative) avec la taille et l'âge des poissons.

De 1971 à 1975, la fécondité relative de la population reproductrice $(\geqslant 2+)$ a varié entre $3650(1973-74)$ et $6110(1972-73)$ ovules/individu (Tableau VI), principalement à cause de l'évolution temporelle des

TABLEAU VI. - Fécondité relative des populations (F.R.P.) reproductrices (mâles et femelles $>2+$ ) de vandoises dans l'Ourthe, de 1971 à 1976.

\begin{tabular}{lccccc}
\hline Années & $\begin{array}{c}\text { F.R.P. } \\
\text { (nombre } \\
\text { d'ovules/individu) }\end{array}$ & 1959 & \multicolumn{4}{c}{$\begin{array}{c}\text { Contribution (\%) } \\
\text { des classes d'âge dominantes } \\
\end{array}$} & 5631 & 5,6 & 3,6 & 63,7 & -1971 \\
\hline $1971-72$ & 6115 & 2,8 & 9,5 & 73,1 & - \\
$1972-73$ & 3655 & 1,7 & 5,0 & 53,3 & 25,3 \\
$1973-74$ & 5595 & - & 4,2 & 47,3 & 35,1 \\
$1974-75$ & 4984 & - & 3,0 & 33,1 & 50,4 \\
$1975-76$ & & & & & \\
\hline
\end{tabular}

structures par âge. Dans tous les cas, 80-90\% de la fécondité de la population est due à un petit nombre de groupes d'âge correspondant à des classes d'âge dominantes.

\subsection{Relations poids - longueur, condition et croissance pondérale}

Les femelles sexuellement mûres sont significativement un peu plus corpulentes que les mâles avant la reproduction (Tableau V) mais à a'autres moments, les différences mâles-femelles sont statistiquement non significatives. Il est de même pour les relations poids-longlieur et la condition des vandoises mûres $(\geqslant 2+)$ et immatures $(0+, 1+$ et $2+<15 \mathrm{~cm}$ ) des deux sexes. Chez ces poissons immatures, le cycle annuel de la condition est peu marqué (maximum en mai-août).

La croissance pondérale annuelle des vandoises de l'Ourthe est décrite par l'équation de Von Bertallanffy suivante:

Poids $t=278\left(1-\mathrm{e}^{-0,33(t-0,24)}\right)^{3,125}$ poids en $g$; âge $t$ en années.

Cette équation est obtenue en combinant le modèle de croissance en longueur ( $c f .1$ ) et une relation poids-longueur standard, log poids (en $\mathrm{g})=-5,153+3,125 \log$ longueur (en $\mathrm{mm}$ ), valable pour les deux sexes réunis et corespondant à la période de développement normal 
des gonades (juillet-septembre). Pour les alevins $0+$ en juillet-novembre, la relation poids-longueur est : $\log$ poids $=-4,971+3,008 \log$ longueur.

\subsection{Evolution d'une cohorte}

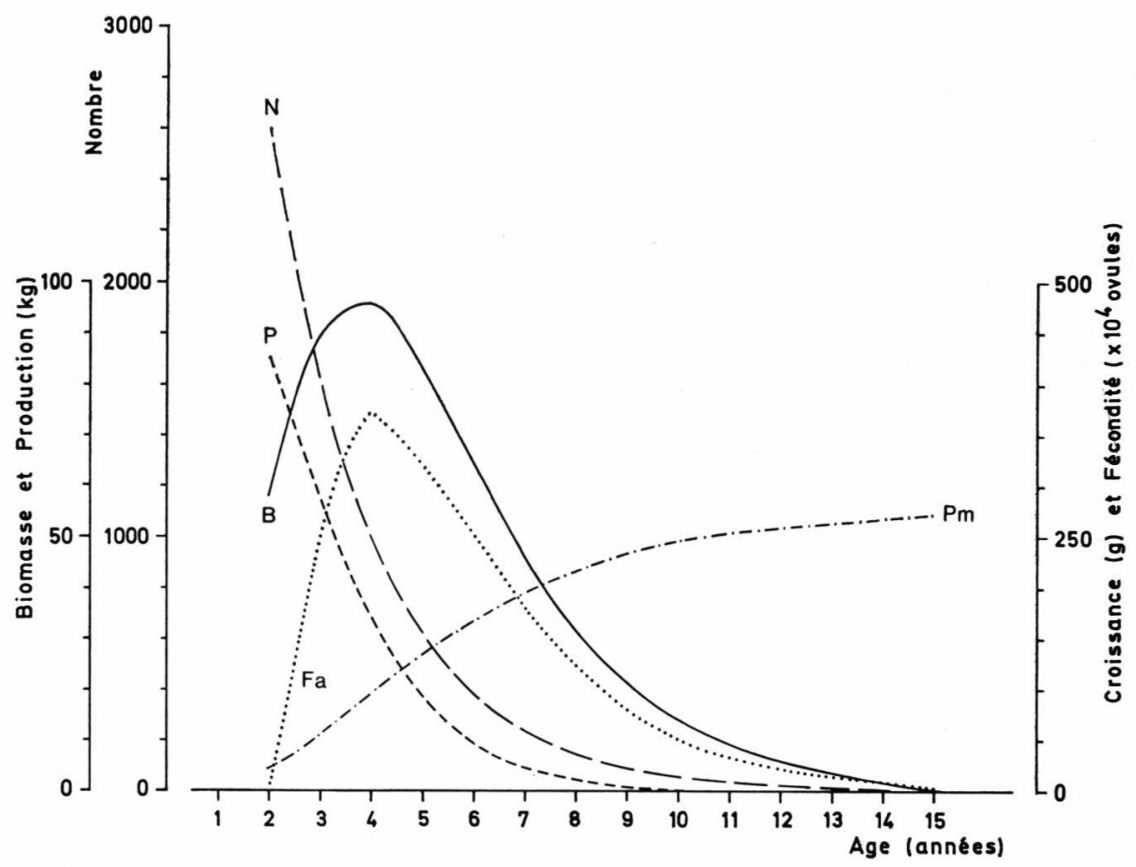

FIG. 6. - Effectifs (N), poids moyen annuel $(\mathrm{Pm})$, biomasse (B), production somatique $(P)$ et fécondité $(\mathrm{Fa})$ d'une cohorte théorique $(1000$ individus à 4 ans) de vandoises (sexes réunis) dans l'Ourthe.

La figure 6 représente l'évolution type d'une cohorte théorique de i 000 vandoises à 4 ans :

- la cohorte atteint sa biomasse maximale à 4 ans (poids et taille critiques de $96 \mathrm{~g}$ et $196 \mathrm{~mm}$ respectivement); la biomasse par recrue à 2 ans est de $218 \mathrm{~g}$;

- la production somatique est de $80 \mathrm{~g} /$ recrue à 2 ans; la courbe de répartition de la production en fonction de l'âge suggère que la production maximale est réalisée pendant les deux premières années, ce qui s'explique par la croissance initiale rapide ;

- la production sexuelle ( $3 \mathrm{~g}, 28 \mathrm{~g}$ et $15 \mathrm{~g} /$ recrue à 2 ans chez les mâles, femelles et sexes réunis respectivement) représente $16 \%$ de la production totale (somatique + sexuelle) d'une cohorte qui est de $95 \mathrm{~g} /$ recrue à 2 ans ; 
- pour les vandoises mâles et femelles de 2 ans et plus, les rapports production somatique/biomasse, production sexuelle/biomasse et production totale/biomasse valent respectivement $0,38,0,07$ et 0,44 .

\subsection{Densité, biomasse, potentiel reprocucteur et production}

En août-octobre 1973, les populations de poissons de l'Ourthe furent ciénombrées par marquage-recapture dans un secteur de $9,6 \mathrm{~km}$ (27,2 ha) (Philippart 1979 b) ; le nombre de vandoises $>10 \mathrm{~cm}$ recapturées était insuffisant $(\mathrm{m}=144 ; \mathrm{c}=234 ; \mathrm{r}=3)$ pour calculer une estimation statistique valable du peuplement. L'ordre de grandeur de celui-ci a été déterminé en multipliant la biomasse capturée pendant les expériences de marquage-recapture $(42,1 \mathrm{~kg}$ soit une population minimale de $1,55 \mathrm{~kg} / \mathrm{ha}$ ) par le coefficient d'efficacité de pêche moyen chez $B$. barbus, $L$. cephalus et $C$. nasus. La biomasse calculée, $6,3 \mathrm{~kg} / \mathrm{ha}$, se rapporte à la population de 1973 presque uniquement composée de vandoises $2+$ (classes 1971) et $5+$ (classe 1967) ; la densité numérique correspondante est d'environ 70 individus $>10 \mathrm{~cm} / \mathrm{ha}$. Des valeurs comparables furent obtenues lors de dénombrements par la méthode des efforts de capture successifs (Seber et Le Cren 1967) dans de courts secteurs $(70-200 \mathrm{~m})$ de l'Ourthe, en 1972-73 et en 1976-79. La population de vandoises forme donc à peine $2 \%$ de l'ichtyomasse totale de l'Ourthe ( $315 \mathrm{~kg} / \mathrm{ha}$, espèces de petite taille exclues).

Avec une biomasse reproductrice de $3,2 \mathrm{~kg} / \mathrm{ha}$ de femelles, le putentiel reproducteur de la population en 1973-74 est estimé à 35 ovules $/ \mathrm{m}^{2}$, soit $0,1 \mathrm{~g} / \mathrm{m}^{2}$ ( $c f .121$ ovules $/ \mathrm{m}^{2}$ et $0,4 \mathrm{~g} / \mathrm{m}^{2}$ dans la Tamise, Mathews 1971). En admettant un rapport $\mathbf{P} / \mathbf{B}$ de 0,4 pour les poissons de 2 ans et plus, on calcule une production totale de $2,8 \mathrm{~kg} / \mathrm{ha} / \mathrm{an}$ comprenant environ $2,3 \mathrm{~kg}$ de production somatique et $0,5 \mathrm{~kg} / \mathrm{ha}$ de production sexuelle; cette estimation ne comprend pas la production des juvéniles $0+$ et $1+$ qui, dans la Tamise (Mathews 1971), contribuent à $62 \%$ de la production totale de la production totale de la population.

Les prélèvements par la pêche estimés en 1974 (2 kg/ha/an) sont équivalents à la production somatique de la fraction réellement exploitée de la population $(\geqslant 2+$ soit $>16 \mathrm{~cm})$.

\subsection{Mouvements}

Les mouvements et la stabilité spatiale n'ont pas été envisagés cians cette étude à cause de la difficulté de réaliser de bons marquages individuels et d'obtenir un nombre suffisant de recaptures. Dans la Tamise, Williams (1965) constate un fort attachement des vandoises à un 
" home site" relativement peu étendu $(250 \mathrm{~m})$ tandis que daris la 'Tweed, Starkie (1976) signale des mouvements de 5-6 $\mathrm{km}$ avec des maxima de $12-13 \mathrm{~km}$ en $2-3$ semaines.

\section{3. - DISCUSSION ET CONCLUSIONS}

Espèce sauvage (non influencée par les rempoissonnements) et euryèce, la vandoise constitue un matériel très intéressant en démographie comparée. Le tableau VII rassemble les principales caractéristiques des populations de vandoises qui ont fait l'objet de recherches approfondies et complètes à ce jour.

Le taux de croissance - de loin le paramètre le mieux connu (Philippart 1971, Hellawell 1974 a, Mann 1974, Hickley et Dexter 1979) - est extrêmement variable selon les milieux : taille moyenne à 5 ans maximale de $210 \mathrm{~mm}$ dans l'Ourthe et minimale de $120 \mathrm{~mm}$ dans la Tamise (Williams 1967, Mathews 1971). Pour 11 populations de vandoises en Grande-Bretagne, Hickley et Dexter (1979) ont calculé une courbe de croissance standard à laquelle correspond une taille moyenne à 5 ans de $185 \mathrm{~mm}$. Les différences de croissance entre les populations sont difficiles à interpréter en l'absence de données précises sur toutes les caractéristiques physico-chimiques, trophiques et biologiques susceptibles d'influencer le taux de croissance. Chez une espèce voisine, Leuciscus cephalus (L.), Mélard (1977) a montré que la température est un facteur clé qui explique la plus grande partie de la variabilité inter-population du taux de croissance.

Dans la plupart des populations connues, les vandoises des deux sexes deviennent sexuellement mûres vers $3-4$ ans; il n'y a pas de relation précise entre l'âge d'acquisition de la maturité et la vitesse de croissance. Mann (1974) suggère que la maturité plus précoce des vandoises de la Frome par rapport à celles de la Stour résulterait de leur croissance plus rapide; le même auteur constate que dans un même groupe d'âge la taille moyenne des vandoises sexuellement mûres est supérieure à celle des vandoises immatures. De même, la maturation plus précoce des mâles pourrait être associée à leur croissance un peu plus rapide que celle des femelles.

En ce qui concerne le sex ratio, la majorité des études révèlent les mêmes caractéristiques : sex ratio général équilibré ou légère prédominance des mâles, prédominance des mâles dans les groupes d'âge inférieurs (jusqu'à 6-7 ans), prédominance des femelles dans les groupes d'âge supérieurs. Hellawell (1974) voit dans cette évolution du sex ratio en fonction de l'âge l'expression d'une réponse différente des 


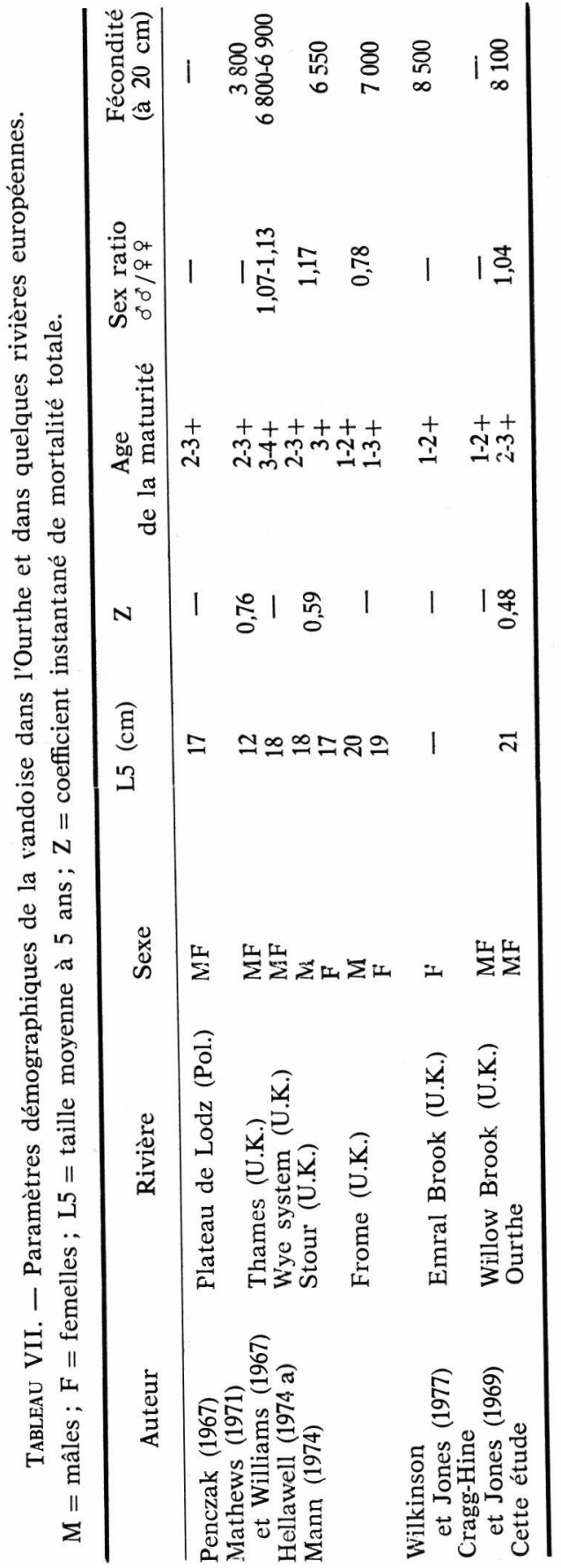


mâles et femelles au stress de la maturité sexuelle et de la reproduction: la maturation sexuelle des femelles et leur participation aux premières reproductions exigeraient des dépenses énergétiques et s'accompagneraient de stress plus importants que chez les mâles; les femelles subiraient donc une mortalité relativement plus importante que les mâles à 3-6 ans (d'où la prépondérance de ceux-ci) mais les individus survivant à cette phase de sélection initiale jouiraient ultéricurement d'une plus grande résistance au stress de la reproduction et d'une plus grande longévité (d'où leur prédominance sur les mâles).

Dans l'Ourthe et dans les rivières étudiées par Mann (1974) et Hellawell (1974) en Grande-Bretagne, on retrouve des caractéristiques fort semblables quant à la biologie de la reproduction de la vandoise : cycle saisonnier de croissance des gonades, poids relatif maximum des gonades et évolution de ce paramètre avec l'âge (augmentation avec l'âge depuis le stade de maturation jusqu'à 6-7 ans puis stabilisation), reproduction en février-mars quand la température de l'eau atteint $7-10^{\circ} \mathrm{C}$. D'après Penczak (1967), la reproduction de la vandoise cans les rivières du plateau de Lodz en Pologne s'étale d'avril à juin ( "spawning of dace is not single») ce qui ne concorde pas avec les situations d'Europe occidentale. Hartley (1947) rapporte que les vandoises sexuellement mûres ( 3 ans et plus) de la rivière Cam en GrandeBretagne ne se reproduisent pas régulièrement chaque année ; ce phénomène d' "intermittent breeding " n'apparaît ni dans l'Ourthe, ni dans la Stour et la Frome (Mann, 1974) et les affluents de la Wye (Hellawell 1974).

La grande variabilité de la fécondité à une taille donnée se superpose à la variabilité des taux de croissance, ce qui suggère l'influence de mêmes facteurs - trophiques ou liés à la densité - sur les deux paramètres.

La fécondité/taille et le taux de croissance élevés observés dans l'Ourthe s'expliquent par des conditions de vie très favorables au point de vue de la qualité de l'eau, de la production secondaire (Micha 1970) et de l'ichtyomasse. Rappelons que, d'après Mackay et Mann (1969), la faible fécondité (croissance) des vandoises de la Tamise résulte d'une insuffisance quantitative (compétition alimentaire) et qualitative (débris végétaux peu énergétiques) des ressources alimentaires; mais dans ce cas, on pourrait aussi envisager un effet de stress dû à la surpopulation (Kipling et Frost 1969).

La dynamique du recrutement dans les populations de vandoises est encore peu connue. L'apparition de mêmes classes d'âge dominantes (1959 et 1964) dans l'Ourthe et dans plusieurs rivières de GrandeBretagne (Cragg-Hine et Jones 1969, Mann 1974, Hellawell 1974 a) confirme le rôle clé des facteurs climatiques et spécialement de la tem- 
pérature (1964 et surtout 1959 ont été des années très chaudes et sèches comme plus récemment 1967 et 1971) dans la détermination du succès de la reproduction. Des études complémentaires sont nécessaires pour vérifier la généralité du phénomène et pour préciser les mécanismes qui interviennent (relation température $\rightarrow$ taux de croissance $\rightarrow$ taux de survie des alevins).

L'extrême irrégularité du recrutement mis en évidence dans la population de vandoises de l'Ourthe est un trait caractéristique c'une espèce se reproduisant dans un environnement climatique assez rude et peu prévisible; les mauvaises conditions abiotiques régnani sur les frayères en mars-avril font échouer la plupart des reproductions mais il suffit d'une conjonction de conditions climatiques très favorables au printemps et en été pour engendrer une classe d'âge ciominante qui assurera la continuité de la population. La maturation sexuelle très précoce des vandoises femelles et leur fécondité relative très élevée par rapport aux autres Cyprinidae rhéophiles lithophiles ( $B$. barbus, $C$. nasus et $L$. cephalus) apparaissent comm? des adaptations démographiques s'inscrivant dans une stratégie de reproduction du type ${ }^{-r}$ (cf. Pianka 1970, Stearns 1977). Des élémenls de cette même stratégie se trouvent aussi chez le hotu, Chondrostoma nasus, qui se reproduit peu de temps après la vandoise (Philippart, sous presse),

Dans les rivières du même type (zone à barbeau selon Huet 1949) ou de la même importance ( $>25 \mathrm{~m}$ de largeur) que l'Ourthe, la vandoise forme rarement des populations contribuant à plus de $5 \%$ de l'ichtyomasse totale : 0,4-1,9 kg/ha dans la Pilica (Penczak et coll., 1976), 1,1-2,3 kg/ha dans la Raba (Kolder et coll., 1974), 4,3 kg/ha, $2,2 \mathrm{~kg} / \mathrm{ha}$, et $26,1 \mathrm{~kg} / \mathrm{ha}$ respectivement pour les groupes $0+, 1+$ et $>2+$ dans la Tamise (Mann 1965, Mathews 1971), 2,7 kg/ha dans la basse Semois (Huet et Timmermans 1963), 12,6 kg/ha en moyenne dans 8 stations de la Semois en 1977 (Philippart $1980 \mathrm{~b}$ ). Une étude en cours sur la répartition géographique et l'état des populations de vandoises dans les rivières de Belgique révèle que des biomasses plus importantes en valeurs absolues (jusqu'à $170 \mathrm{~kg} / \mathrm{ha}$ dañs un échantillon de 17 stations du bassin de l'Ourthe où la biomasse moyenne est de $21,9 \mathrm{~kg} / \mathrm{ha}$; Philippart $1980 \mathrm{a}$ ) et en valeur relative (25-40\% de l'ichtyomasse) apparaissent dans certaines petites rivières (zone à ombre et à barbeau) où la biomasse des Cyprinidae rhéophiles de grande taille (Barbus barbis, Chondrostoma nasus et Leuciscus cephalus) est faible, soit naturellement, soit à cause d'une altération de la qualité de l'eau (pollution modérée) ou du milieu (chenalisation). Dans ces milieux altérés, la mcdification structurelle de l'ichtyofaune se traduit généralement par une forte régression des espèces normalement présentes (Barbus barbus et Chondrostoma 
nasus) et leur remplacement par des espèces plus résistantes : Leuciscus leuciscus (qui tend à remplacer $C$. nasus) et Leuciscus cephalus (qui tend à remplacer $B$. barbus). Nos recherches actuelles ont pour objectif de définir quantitativement les facteurs influençant la répartition géographique et la biomasse des populations de vandoises et d'analyser les phénomènes de remplacement d'espèces.

Enfin, pour ce qui concerne la production écologique, il y a peu de connées comparatives. Dans la Tamise (Mathews 1971), la production des vandoises de 2 ans et plus (biomasse : $26,1 \mathrm{~kg} / \mathrm{ha}$ ) est trois fois plus importante $(10,6 \mathrm{~kg} / \mathrm{ha})$ que dans l'Ourthe mais les rapports $\mathrm{P} / \mathrm{B}$ sont fort semblables $(0,45)$. Dans une section polluée de la rivière Pilica en Pologne, Penczak et coll. (1978) calculent des productions de 1,0 à $1,4 \mathrm{~kg} / \mathrm{ha}$, ce qui est comparable à l'Ourthe. Des productions plus importantes doivent toutefois être attendues dans les cours d'eau où le peuplement en vandoises atteint des valeurs plus élevées.

\section{REMERCIEMENTS}

Cette étude a été réalisée grâce à des mandats et crédits (S 2/5-F4-88110-D) de recherche du Fonds National de la Recherche Scientifique (F.N.R.S.) et grâce à cies subsides de fonctionnement de la Commission provinciale de Liège du Fonds piscicole (Ministère de l'Agriculture) et de la section belge PF (Production Freshvaters) du Programme Biologique International. Les autorisations de pêche à l'électricité ont été accordées par l'Administration des Eaux et Forêts. Pour !'étude sur le terrain, nous avons bénéficié de l'appui logistique de l'Aquarium de l'Institut de Zoologie de l'Université de Liège ( $\mathrm{D}^{\mathrm{r}} \mathrm{J}$. Voss).

\section{TRAVAUX CITÉS}

Backiel (T.). 1971. - Production and food consumption of predatory fish in the Vistula river. J. Fish. Biol., $3: 369-405$.

Blanc (M.), Banarescu (P.), Gaudet (J.L.) et Hureau (J.C.). 1971. - Poissons des eaux continentales d'Europe. Catalogue multilingue. Fishing News Books Etd, London.

Bourgeois (M.). 1963. - La vandoise. La Pêche et les Poissons, 219 : 39.

Broughton (N.M.) et Jones (N.V.). 1978. - An investigation into the growth of 0 - group roach (Rutilus rutilus L.) with special reference to temperature. J. Fish. Biol., 12 : 345-357.

Chapman (D.W.). 1968. - Production, pp. 199-214. In W.E. Ricker Ed., Methods for assessment of fish production in freshwater, Blackwell Scientific Publication, Oxford et Edinbourgh : 339 p.

CragG-Hine (D.) et Jones (J.W.). 1969. - The growth of dace Leuciscus leuciscus (L.), roach Rutilus rutilus (L.) and chub Leuciscus cephalus (L.) in Willow Brook, Northamptonshire. J. Fish. Biol., 1 : 59-82.

HART (P.J.B.) et Pitcher (T.J.). 1969. - Field trials of fish marking using a jet inoculator. J. Fish. Biol., 1 (4) : 383-385. 
HaRT (P.J.B.) et Pitcher (T.J.). 1973. - Population densities and growth of fish in the River Nene, Northamptonshire. J. Inst. Fish. Mgmt., 4 (3) : 69-86.

Hellawell (J.M.). 1974 a. - The ecology of populations of dace, Leuciscus leuciscus (L.) from two tributaries of the river Wye, Herefordshire, England. Freshwat. Biol., 4 (6) : 557-604.

Hellawell (J.M.). 1974 b. - The problems of protracted cheek formation and the validity of the use of scales in age determination examplified by two populations of dace Leuciscus leuciscus (L.), pp. 173-180, In T.B. Bagenal (Ed.), Ageing of Fish, Unwin Brothers Ltd, 234 p.

HuEt (M.). 1949. - Aperçu des relations entre la pente et les populations pis. cicoles des eaux courantes. Revue Suisse d'Hydrologie, 11 (3-4) : 332-351.

Huet (M.) et Timmermans (J.A.). 1963. - La population piscicole de la Semois inférieure, grosse rivière belge du type supérieur de la zone à barbeau. Trav. Stat. Rech. Eaux et Forêts, Groenendaal, série D, n ${ }^{\circ} 36: 1-31$.

HICKLEY (P.) et DesTER (K.F.). 1979. - A comparative index for quantifying growth in length in fish. Fish Mgmt., 10 (4) : 147-151.

HoFstede (A.E.). 1974. - Studies on growth, ageing and back calculation of roach Rutilus rutilus (L.) and dace Leuciscus leuciscus (L.), pp. 137-147, In T.B. Bagenal (Ed.), The Ageing of Fish, Unwin Brothers Ltd, 234 p.

KEMPE (O.). 1962. - The growth of roach (Leuciscus rutilus L.) in some Swedish Lakes. Rep. Inst. Freshwat. Res. Drotiningholm, 44 : 42-104.

Kennedy (M.). 1969. - Spawning and early development of the dace Leuciscus leuciscus (L.). J. Fish Biol., 1 : 249-259.

Kipling (C.) et Frost (W.E.). 1969. - Variations in the fecundity cf pike, Esox lucius L. in Windermere. J. Fish Biol., $1: 221-237$.

KLIMczYK (M.). 1966. - Der Hasel (Leuciscus leuciscus (L.)) aus dem Fluss Czarna Staszowska. Acta Hydrobiol., Krakow, 8, 347-370.

KlimczyK-Janikowska (M.). 1971. - Dace, Leuciscus leuciscus (L.) from the upper Vistula and Czarna Przemsza. Acta Hydrobiol., Krakow, $13: 343-361$.

KOLdER (W.), SKora (S.) et Wlodek (J.M.). 1974. - Ichthyofauna of the river Raba and of its tributaries. Acta Hydrobiol., Krakow, $16: 65-99$.

LE CREN (E.D.). 1951. - The length - weight relationship and seasonal cycle in gonad weight and condition in the perch (Perca fluviatilis). J. Anim. Ecol., 30 : 201-219.

MackaY (I.) et MANN (K.H.). 1969. - Fecundity of two Cyprinid fishes in the River Thames, Reading, England. J. Fish. Res. Board Can., 26 : 2795-2805.

MaNN (R.K.H.). 1973. - Observation on the age, growth, reproduction and food of the roach (Rutilus rutilus (L.)) in two rivers in Southern England. $J$. Fish Biol., 5 : 707-736.

MANN (R.K.H.). 1974. - Observation on the age, growth, reproduction and food of the dace, Leuciscus leuciscus (L.) in two rivers in Southern England. J. Fish Biol, 6 : 237-254.

Mathews (C.P.). 1971. - Contribution of young fish to total production of fish in the River Thames near Reading. J. Fish Biol., $4: 157-180$.

Mathews (C.P.) et Williams (W.P.). 1972. - Growth and annual check formation in scales of dace, Leuciscus leuciscus (L.). J. Fish Biol., $4: 363-367$.

Melard (Ch.). 1977. - Analyse des facteurs influençant la croissance du chevaine (Leuciscus cephalus (L.)) en rivière. Mémoire de Licence en Sciences zoologiques, Université de Liège, $52 \mathrm{p}$.

Micha (J.C.). 1970. - Etude quantitative du benthos d'une rivière de Belgique : l'Ourthe liégeoise. Annls Limnol., 6 (3) : 255-280.

Micha (J.C.). 1971. - Etude des communautés piscicoles de l'Ourthe liégeoise. Tribune du CEBEDEAU, $326: 1-8$.

MILLS (C.A.). 1980. - Spawning and rearing eggs of dace Leuciscus leuciscus (L.) Fish. Mgmt., 11 (2): 67-72. 
Philippart (J.C.). 1971. - Age et croissance de la vandoise, Leuciscus leuciscus (L.) dans l'Ourthe et la Berwine. Ann. Soc. Roy. Zool. Belg., 103 (1) : 61-77.

Philippart (J.C.). 1977. - Contribution à l'étude de l'écosystème "rivière de la zone à Barbeau": Densité, biomasse et production des populations de poissons de l'Ourthe, pp. 551-567, In P. Duvigneaud et P. Kestemont (Ed.), Productivité biologique en Belgique, Duculot, Gembloux, 617 p.

Philippart (J.C.). 1979 a. Introduction à l'étude des aspects écologiques et socioéconomiques de la pêche récréative. Enquête sur la pêche récréative dans l'Ourthe à Hamoir. Bulletin de la Société géographique de Liège, 15 : 229250.

Philippart (J.C.). 1979 b. - Observation concernant l'efficacité de la pêche à l'électricité dans une rivière de la zone à barbeau. Bull. fr. Pisc., 273 : 157172.

Philippart (J.C). 1980 a. - Essai d'évaluation des ressources ichtyologiques dans le bassin de l'Ourthe (bassin de la Meuse) en Belgique. Consultation technique de la CECPI (Commission Européenne Consultative pour les Pêches dans les Eaux Intérieures), 20-24 avril 1980, Vichy, France, 34 p.

Philippart (J.C.). $1980 \mathrm{~b}$. - Incidences de la pollution organique et de l'eutrophisation sur la faune ichtyologique de la Semois. Annls Limnol., (16) 1 : 77-89.

PhilipPart (J.C.). 1981. - Ecologie d'une population de chevaines, Leuciscus cephalus (L.) (Pisces: Cyprinidae) dans la rivière Ourthe en Belgique. Communication présentée à la séance du 14 mars 1981 de la Société Royale Zoologique de Belgique sur le thème: Etudes de populations animales.

Philippart (J.C.). (Sous presse). - Démographie du hotu, Chondrostoma nasus (L.) (Pisces : Cyprinidae) dans l'Ourthe (bassin de la Meuse, Belgique). Ann. Soc. roy. Zool. Belg.

Penczak (T.). 1967. - The dace, Leuciscus leuciscus (L.) from the Lodz Upland and adjacent areas. Part I. Material for the knowledge of the biology of the dace. Acta Hydrobiol. Krakow, $9: 281-335$.

Penczak (T.). 1979. - Ecological fish production in polish rivers, pp. 11-29, in Proceedings of the First British Freshwater Fisheries Conference, The University of Liverpool, $385 \mathrm{p}$.

Penczak (T.), Zalewski (M.) et Pfeifer (K.). 1978. - Materials for the ecology of the dace, Leuciscus leuciscus (L.) in the barbel region of the polluted Pilica River. 1. Production and food consumption. Acta Hydrobiol., 20 : 63-85.

Pianka (E.R.). 1970. - On r - and K - selection. Amer. Nat., 104 : 592-597.

RICKER (W.E.). 1968. - Methods for assessment of fish production in fresh waters. IBP Handbook $n^{\circ} 3$, Blackwell Scientific Publications, Oxford and Edinburg, 313 p.

SEBER (G.A.F.) et LE CREN (F.D.). 1967. - Estimating population parameters from catches large relative to the population. J. anim. Ecol., $36: 631-643$.

Starkie (A.). 1976. - Ecology of dace in river Tweed. Fish. Mgmt., 7 (4) : 88.

StEARNS (S.C.). 1977. - The evolution of life history traits : a critique of the theory and a review of the data. Ann. Rev. Ecol. Syst., $8: 145-171$.

Wilkinson (D.R.) et Jones (J.W.). 1977. - The fecundity of dace, Leuciscus leuciscus (L.) in Emrel Brook, Clwynd, North Wales. Fresh. Biol., 7 (2) : 135-145.

Wrlliams (W.P.). 1965. - The population density of four species of freshwater fish, roach (Rutilus rutilus L.), bleak (Alburnus alburnus L.), dace (Leuciscus leuciscus (L.) and perch (Perca fluviatilis L.) in the River Thames at Reading. J. Anim. Ecol., 34, 173-185.

Williams (W.P.). 1967. - The growth and mortality of four species of fish in the River Thames at Reading. J. Anim. Ecol., 36 : 695-720. 